Volume 2, Issue 2, pages 169-178

p-ISSN 2655-8564, e-ISSN 2685-9432

\title{
The Lagrangian and Hamiltonian for RLC Circuit: Simple Case
}

\author{
Albertus Hariwangsa Panuluh \\ Department of Physics Education, Faculty of Teacher Training and \\ Education, Sanata Dharma University, Yogyakarta, Indonesia \\ Corresponding Author: panuluh@usd.ac.id
}

(Received 11-04-2020; Revised 06-06-2020; Accepted 12-06-2020)

\begin{abstract}
The Lagrangian and Hamiltonian for series RLC circuit has been formulated. We use the analogical concept of classical mechanics with electrical quantity. The analogy is as follow mass, position, spring constant, velocity, and damping constant corresponding with inductance, charge, the reciprocal of capacitance, electric current, and resistance respectively. We find the Lagrangian for the LC, RL, RC, and RLC circuit by using the analogy and find the kinetic and potential energy. First, we formulate the Lagrangian of the system. Second, we construct the Hamiltonian of the system by using the Legendre transformation of the Lagrangian. The results indicate that the Hamiltonian is the total energy of the system which means the equation of constraints is time independent. In addition, the Hamiltonian of overdamping and critical damping oscillation is distinguished by a certain factor.
\end{abstract}

Keywords: RLC circuit, Lagrangian, Hamiltonian, Legendre transformation 


\section{International Journal of Applied Sciences and Smart Technologies}

Volume 2, Issue 2, pages 169-178

p-ISSN 2655-8564, e-ISSN 2685-9432

\section{Introduction}

Lagrangian and Hamiltonian are topics part of analytical mechanics or classical mechanicscourse that s studied in the second or third year of the undergradute physics program.Some advantages are obtained when using Lagrangian and Hamiltonian formalismto solve the mechanical problems. The Lagrangian and Hamiltonian formalism solve mechanicalproblems using the energy of the system, which is a scalar quantity. Differentfrom Newtonian mechanics which solve the mechanical problem using force, which is avector quantity [1].

The RLC circuit is studied as part of the electricity and magnetism course by physics students as well as physics education students. The problem discussed in physics textbooks about the properties of electric currents and the potential difference or voltage that are in the electronic devices on the circuit [2]. Moreover, the RLC circuit becomes an example in the differential equations subtopics in the mathematical physics course [3]. However, the RLC circuit is not only limited to the topic of electricity or solving differential equations. Current RLC research is now heading toward the quantum level. This is due to the rapid development of nanotechnology so that nanosized electronic devices are being developed. Theoretical research about nanoelectronic science has been conducted and has result in quantum transport, computational nanoelectronics, new device concepts, and novel transport physical phenomena found in small structures [4]. The quantum mechanics of the RLC circuit which obtained the approximation of energy eigenvalues in terms of a dimensionless parameter has also been studied [5].

One of the methods to quantize the RLC circuit is using the Hamiltonian formalism. For example, using Cardirola-Kanai Hamiltonian and quantum invariant method to solve the Schrdinger equation for the RLC circuit [6]. For advance, there is also a research quantum mechanical effect of the underdamped, critically damped, and overdamped electric circuits with a power source using a time dependent Hamiltonian operator [7].

This research aims to formulate the Hamiltonian extension for the series RLC circuit without emf source, which is the simple case. We formulate the Hamiltonian by using 


\section{International Journal of Applied Sciences and Smart Technologies}

Volume 2, Issue 2, pages 169-178

p-ISSN 2655-8564, e-ISSN 2685-9432

the Legendre transformation of the Lagrangian from [8]. In addition, for educational purpose, this research enriches classical mechanics learning materials based on the lecturer' research.

\section{Reasearch Methodology}

We formulate the Hamiltonian theoretically based on the Lagrangian from [8]. First, we formulate the Lagrangian of series RLC circuits by using the analogy between classical mechanics with electrical quantity [9]. These analogies are shown in Table 1.

Table 1. Analogy between mechanical quantity and electrical quantity

\begin{tabular}{cc}
\hline Mechanical Quantity & Electrical Quantity \\
\hline mass $(m)$ & inductance $(L)$ \\
position $(x)$ & charge $(Q)$ \\
spring constant $(k)$ & reciprocal of capacitance $(1 / C)$ \\
velocity $(v)$ & electric current $(I)$ \\
damping constant $(\beta)$ & resistance $(R)$ \\
\hline
\end{tabular}

Second, we construct the Hamiltonian of the system by using the Legendre transformation of the Lagrangian. After that, we check the Hamiltonian whether it is the total energy of the system or not. The limitation of this research is the series electric circuit without emf (electric source). That is why this research is a simple case.

\section{Results and Discussion}

In this section, we present our research results and discussion about Lagrangian and Hamiltonian approaches for the problem.

\subsection{Lagrangian}

Here we will formulate the Lagrangian of the series electric circuit, such as: LC, RL, $\mathrm{RC}$, and RLC circuit.

\section{LC Circuit}

To formulate the Lagrangian, first, we have to solve the differential equation of Kirchhoffs rule for series LC circuit without emf source as follow 


\section{International Journal of Applied Sciences and Smart Technologies}

Volume 2, Issue 2, pages 169-178

p-ISSN 2655-8564, e-ISSN 2685-9432

$$
\frac{d^{2} q}{d t^{2}}+\frac{q}{L C}=0
$$

which has a solution for discharging capacitor

$$
q=Q_{0} e^{-i \omega t}
$$

with $Q_{0}$ is initial capacitance and $\omega$

$$
\omega=\sqrt{\frac{1}{L C}} .
$$

To find the Lagrangian of the system, we first look for kinetic and potential energy. We use the analogy in Table 1, so the kinetic energy for LC circuit (TLC) is

$$
T_{L C}=\frac{1}{2} L \dot{q}^{2}=-\frac{1}{2} L \omega^{2} Q_{0}^{2} e^{-2 i \omega t},
$$

and the potential energy for LC circuit (VLC) is

$$
V_{L C}=\frac{1}{2 C} q^{2}=\frac{1}{2 C} Q_{0}^{2} e^{-2 i \omega t} .
$$

Therefore, the Lagrangian (we use L for Lagrangian notation to prevent confusion with inductance symbol) is

$$
L_{L C}=-\frac{1}{2} Q_{0}^{2} e^{-2 i \omega t}\left(L \omega^{2}+\frac{1}{C}\right)
$$

\section{RL Circuit}

We assume that the initial current that flows in the RL circuit is I0. Then, the differential equation for the series RL circuit is

$$
L \frac{d I}{d t}+R I=0
$$

which has a solution

$$
I=I_{0} e^{-R t / L}
$$

We know that current is the derivative of charge to time which in mechanical quantity analog to velocity $(v)$. So, the kinetic energy for RL circuit is

$$
T_{R L}=\frac{1}{2} L I_{0}^{2} e^{-2 R t / L},
$$

and we assume that RL circuit does not have potential energy because there is no 


\section{International Journal of Applied Sciences and Smart Technologies}

Volume 2, Issue 2, pages 169-178

p-ISSN 2655-8564, e-ISSN 2685-9432

capacitance. Therefore, the Lagrangian for the RL circuit is

$$
L_{R L}=\frac{1}{2} L I_{0}^{2} e^{-2 R t / L} .
$$

\section{RC Circuit}

The differential equation for the series $\mathrm{RC}$ circuit with $Q_{-} 0$ as the initial charge of the capacitor is

$$
R \frac{d q}{d t}+\frac{q}{C}=0
$$

which has a solution

$$
q=Q_{0} e^{-t / R C} .
$$

We know that the kinetic energy is $1 / 2 m v^{2}$ and from Table 1 mass has analogy as inductance $(L)$. However, because there is no inductance in the RC circuit, the RC circuit does not have kinetic energy. The potential energy for RC circuit is

$$
V_{R C}=\frac{1}{2 C} Q_{0}^{2} e^{-2 t / R C} \text {. }
$$

Therefore, the Lagrangian for the RC circuit is

$$
L_{R C}=\frac{1}{2 C} Q_{0}{ }^{2} e^{-2 t / R C} .
$$

\section{RLC Circuit}

The differential equation for series RLC circuit is

$$
\frac{d^{2} q}{d t^{2}}+\frac{R}{L} \frac{d q}{d t}+\frac{q}{L C}=0
$$

This equation is analog with damped harmonic oscillation. Then we define

$$
\gamma=\frac{R}{2 L}
$$

and

$$
\omega_{0}=\sqrt{\frac{1}{L C}},
$$

where $\gamma$ and $\omega_{0}$ as damping factor and natural frequency respectively. So equation (15) can be written as 


\section{International Journal of Applied Sciences and Smart Technologies}

Volume 2, Issue 2, pages 169-178

p-ISSN 2655-8564, e-ISSN 2685-9432

$$
\frac{d^{2} q}{d t^{2}}+2 \gamma \frac{d q}{d t}+\omega_{0}^{2} q=0,
$$

which has a general solution

$$
q=K_{1} e^{-(\gamma-\lambda) t}+K_{2} e^{-(\gamma+\lambda) t},
$$

where

$$
\lambda=\sqrt{\gamma^{2}-\omega_{0}^{2}} .
$$

There are three possibilities: overdamping, critical damping, and underdamping.

\section{a) Overdamping}

The overdamping will occur if $\lambda>0$. Equation (19) for the overdamping case is

$$
q=Q_{0} e^{-(\gamma+\lambda) t}
$$

where $Q_{0}$ is the initial charge in the capacitor. The kinetic energy is

$$
T_{\text {over }}=\frac{1}{2} L(\gamma+\lambda)^{2} Q_{0}^{2} e^{-2(\gamma+\lambda) t},
$$

and the potential energy is

$$
V_{\text {over }}=\frac{1}{2 C} Q_{0}^{2} e^{-2(\gamma+\lambda) t} .
$$

Therefore, the Lagrangian for the overdamping case is

$$
\bar{L}_{\text {over }}=\frac{1}{2 C} Q_{0}^{2} e^{-2(\gamma+\lambda) t}\left[L(\gamma+\lambda)^{2}-\frac{1}{C}\right] .
$$

\section{b) Critical damping}

The condition for critical damping oscillation is $\lambda=0$. Then equation (19) for critical damping case is

$$
q=Q_{0} e^{-\gamma t}
$$

The kinetic energy is

$$
T_{\text {crit }}=\frac{1}{2} L \gamma^{2} Q_{0}^{2} e^{-2 \gamma t},
$$

and the potential energy is 


\section{International Journal of Applied Sciences and Smart Technologies}

Volume 2, Issue 2, pages 169-178

p-ISSN 2655-8564, e-ISSN 2685-9432

$$
V_{\text {crit }}=\frac{1}{2 C} Q_{0}^{2} e^{-2 \gamma t}
$$

So, the Lagrangian for the critical damping case is

$$
\bar{L}_{\text {crit }}=\frac{1}{2} Q_{0}^{2} e^{-2 \gamma t}\left(L \gamma^{2}-\frac{1}{C}\right) \text {. }
$$

\section{c) Under damping}

The under damping will occur if $\lambda<0$. Then equation (19) for under damping case is

$$
q=e^{-\gamma t}\left(A \cos \left(\alpha t-\theta_{0}\right)\right)
$$

where

$$
\alpha=\sqrt{\omega_{0}^{2}-\gamma^{2}}=\sqrt{\frac{1}{L C}-\frac{R^{2}}{4 L^{2}}} .
$$

We choose $\theta_{0}=0^{\circ}$ and $A=Q_{0}$ then equation (29) becomes

$$
q=e^{-\gamma t} Q_{0} \cos (\alpha t) .
$$

The kinetic energy is

$$
T_{\text {under }}=\frac{1}{2} L Q_{0}^{2} e^{-2 \gamma t}[\gamma \cos (\alpha t)+\alpha \sin (\alpha t)]^{2},
$$

and the potential energy is

$$
V_{\text {under }}=\frac{1}{2 C} e^{-2 \gamma t} Q_{0}^{2} \cos ^{2}(\alpha t) .
$$

Therefore, the Lagrangian for under damping case is

$$
\bar{L}_{\text {under }}=\frac{1}{2} Q_{0}^{2} e^{-2 \gamma t}\left[L[\gamma \cos (\alpha t)+\alpha \sin (\alpha t)]^{2}-\frac{1}{C} \cos ^{2}(\alpha t)\right] .
$$

\subsection{Hamiltonian}

In this subsection, we will derive the Hamiltonian of each electric circuit. Hamiltonian can be formulated by using Legendre transformation from Lagrangian as follow [10] 


\section{International Journal of Applied Sciences and Smart Technologies}

Volume 2, Issue 2, pages 169-178

p-ISSN 2655-8564, e-ISSN 2685-9432

$$
H=\sum_{\alpha=1}^{n} p_{\alpha} \dot{q}_{\alpha}-L\left(q_{1}, \ldots, q_{\alpha}, \dot{q}_{1}, \ldots, \dot{q}_{\alpha}, t\right),
$$

where $p_{\alpha}$ and $q_{\alpha}$ are the generalized momenta and generalized coordinates respectively. After some calculations, we find the Hamiltonian for all electric circuit are as follows:

$$
\begin{gathered}
H_{L C}=\frac{1}{2} Q_{0}^{2} e^{-2 i \omega t}\left(L \omega^{2}\right), \\
H_{R L}=\frac{1}{2} L I_{0}^{2} e^{-2 R t / L}, \\
H_{R C}=\frac{1}{2 C} Q_{0}{ }^{2} e^{-2 t / R C}, \\
H_{\text {over }}=\frac{1}{2} Q_{0}^{2} e^{-2(\gamma+\lambda) t}\left[L(\gamma+\lambda)^{2}+\frac{1}{C}\right], \\
H_{\text {crit }}=\frac{1}{2} Q_{0}^{2} e^{-2 \gamma t}\left[L \gamma^{2}+\frac{1}{C}\right], \\
H_{\text {under }}=\frac{1}{2} Q_{0}^{2} e^{-2 \gamma t}\left[L[\gamma \cos (\alpha t)+\alpha \sin (\alpha t)]^{2}-\frac{1}{C} \cos ^{2}(\alpha t)\right] .
\end{gathered}
$$

\subsection{Discussions}

This research aims to formulate the Hamiltonian extension for the series RLC circuit without emf source. So this research is the simple case because without emf source. If we include the emf source, the equation become the driven case with the emf source become the driven factor [11]. From the results above, we find that the Hamiltonian for LC, RL, RC, and RLC circuits (equations (36)-(41)) are the total energy of the systems. That is kinetic energy plus potential energy. We also find that the Hamiltonian of the RL circuit is the same as the Lagrangian. The Hamiltonian of overdamping and critical 


\section{International Journal of Applied Sciences and Smart Technologies}

Volume 2, Issue 2, pages 169-178

p-ISSN 2655-8564, e-ISSN 2685-9432

damping is distinguished by a certain factor. For further research, we can quantize the RLC circuit by using the Poisson bracket and using these Hamiltonians.

In addition, this simple case research can be easily followed by students that learn analytical mechanics. In Lagrange and Hamilton mechanics learning material, the students usually deal with complex mechanics problem. With this research, students know that Lagrange and Hamilton mechanics are not only used to solve mechanical problems. This also can be used to solve in the case of electricity problems. So this research can be an additional material in analytic mechanics course.

\section{Conclusion}

This research aims to formulate the Hamiltonian extension for the series RLC circuit without emf source. So this research is the simple case because without emf source. If we include the emf source, the equation become the driven case with the emf source become the driven factor [11]. From the results above, we find that the Hamiltonian for LC, RL, RC, and RLC circuits (equations (36)-(41)) are the total energy of the systems. That is kinetic energy plus potential energy. We also find that the Hamiltonian of the RL circuit is the same as the Lagrangian. The Hamiltonian of overdamping and critical damping is distinguished by a certain factor. For further research, we can quantize the RLC circuit by using the Poisson bracket and using these Hamiltonians.

In addition, this simple case research can be easily followed by students that learn analytical mechanics. In Lagrange and Hamilton mechanics learning material, the students usually deal with complex mechanics problem. With this research, students know that Lagrange and Hamilton mechanics are not only used to solve mechanical problems. This also can be used to solve in the case of electricity problems. So this research can be an additional material in analytic mechanics course.

\section{Acknowledgement}

The author thanks LPPM USD for the research funds. 


\section{International Journal of Applied Sciences and Smart Technologies}

Volume 2, Issue 2, pages 169-178

p-ISSN 2655-8564, e-ISSN 2685-9432

\section{References}

[1] G. R. Fowles and G. L. Cassiday, Analytical Mechanics, Thomson Learning, Belmont, 7th Edition, 2005.

[2] R. A. Serway and J. W. Jewett, PHYSICS for Scientists and Engineers with Modern Physics, Thomson Learning, Belmont, 7th Edition, 2008.

[3] M. L. Boas, Mathematical Methods in the Physical Sciences, John Wiley \& Sons, New Jersey, 3rd Edition, 2006.

[4] F. A. Buot, "Mesoscopic physics and nanoelectronics: nanoscience and nanotechnology." Physics Report, 234 (2-3), 73-174, 1993.

[5] C. A. U. Daz, "Discrete-charge quantum circuits and electrical resistance." Physics Letters A, 372 (30), 5059-5063, 2008.

[6] I. A. Pedrosa and A. P. Pinheiro, "Quantum description of a mesoscopic RLC circuit.” Progress of Theoretical Physics, 125 (6), 1133-1141, 2011.

[7] J. R. Choi, "Quantization of underdamped, critically damped, and overdamped electric circuits with a power source." International Journal of Theoretical Physics, 41 (10), 1931-1939, 2002.

[8] A. H. Panuluh and A. Damanik, "Lagrangian for RLC circuits using analogy with the classical mechanics concepts." Journal of Physics: Conference Series, 909, 012005, 2017.

[9] H. Essen, "From least action in electrodynamics to magnetomechanical energy - a review.” European Journal of Physics, 30 (3), 515-539, 2009.

[10]A. P. Arya, Introduction to Classical Mechanics, Prentice-Hall, New Jersey, 2nd Edition, 1998.

[11]K. Ozdas and M. Kilickaya, "Novel method for the analysis of RLC circuits." International Journal of Electronics Theoretical and Experimental, 70 (2), $407-$ 412, 1991. 